\title{
Physalin D inhibits RANKL-induced osteoclastogenesis and bone loss via regulating calcium signaling
}

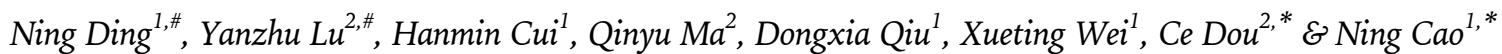 \\ ${ }^{1}$ Department of Blood Purification, General Hospital of Shenyang Military Area Command, Shenyang 110000, ${ }^{2}$ Department of Orthopedics, \\ Southwest Hospital, Third Military Medical University (Army Medical University), Chongqing 400038, China
}

\begin{abstract}
We investigated the effects of physalin A, B, D, and F on osteoclastogenesis induced by receptor activator of nuclear factor $\kappa B$ ligand (RANKL). The biological functions of different physalins were first predicted using an in silico bioinformatic tool (BATMAN-TCM). Afterwards, we tested cell viability and cell apoptosis rate to analyze the cytotoxicity of different physalins. We analyzed the inhibitory effects of physalins on RANKL-induced osteoclastogenesis from mouse bone-marrow macrophages (BMMs) using a tartrate-resistant acid phosphatase (TRAP) stain. We found that physalin D has the best selectivity index (SI) among all analyzed physalins. We then confirmed the inhibitory effects of physalin D on osteoclast maturation and function by immunostaining of F-actin and a pit-formation assay. On the molecular level, physalin D attenuated RANKLevoked intracellular calcium $([\mathrm{Ca}(2+)](\mathrm{i}))$ oscillation by inhibiting phosphorylation of phospholipase C $\gamma 2$ (PLC $\gamma 2)$ and thus blocked the downstream activation of $\mathrm{Ca} 2+/$ calmodulindependent protein kinases (CaMK)IV and cAMP-responsive element-binding protein (CREB). An animal study showed that physalin $D$ treatment rescues bone microarchitecture, prevents bone loss, and restores bone strength in a model of rapid bone loss induced by soluble RANKL. Taken together, these results suggest that physalin D inhibits RANKL-induced osteoclastogenesis and bone loss via suppressing the PLC $\gamma 2$-CaMK-CREB pathway. [BMB Reports 2020; 53(3): 154-159]
\end{abstract}

\section{INTRODUCTION}

In human body, the homeostasis of bone is maintained by

*Corresponding authors. Ning Cao, Tel: +86-24-28851193; E-mail: szxyjh@aliyun.com; Ce Dou, Tel: +86-23-65340297; E-mail: lance. douce@gmail.com

${ }^{\#}$ These authors contributed equally to this work.

https://doi.org/10.5483/BMBRep.2020.53.3.147

Received 27 May 2019, Revised 11 June 2019, Accepted 29 August 2019

Keywords: Bone, Calcium Signaling, Osteoclast, Osteoporosis, Physalin constant remodeling that was conducted by osteoclastic bone resorption and osteoblastic bone formation. In adulthood, osteoclasts are the only cell type for bone resorption that is derived from hematopoietic stem cells. Hematopoietic cytokines including macrophage-colony stimulating factor (M-CSF, encoded by CSF1) and receptor activator of nuclear factor $\kappa B$ ligand (RANKL) are two most important stimulating factors for osteoclastogenesis (1). Although M-CSF mainly maintains the proliferation and survival of preosteoclasts, RANKL stimulates the differentiation and maturation of preosteoclasts. Upon stimulation by RANKL, RANK activation initiates downstream TNF receptor-associated factor 6 (TRAF6) signaling and further induces the expression of nuclear factor of activated T cells c1 (NFATc1), the key regulator in osteoclast differentiation $(2,3)$. Osteoclast dysfunction occurs in various congenital or acquired bone disorders such as, osteoporosis, osteopetrosis, Paget's disease and osteoarthritis $(4,5)$.

Physalis alkekengi L. is a perennial herbaceous plant that is widespread in Europe, northeast Asia, and southwest Asia. Traditionally, this herb is considered to be antidiuretic, anti-inflammatory, antipyretic, and antinociceptive (6, 7). Biological active extracts isolated from Physalis species are called physalins, which have been reported regulatory in immune system $(8,9)$. Moreover, anti-inflammatory, antimalarial, and antinociceptive effects of physalins have been reported (10-12). We also previously reported that Physalin D can regulate macrophage polarization towards a M2 phenotype (13). However, the effects of physalin in the skeletal system have not been reported, whereas that physalin was suppressive in macrophage activation has been (7). Given the fact that osteoclasts share the same myeloid cell lineage as do macrophages, it is plausible to hypothesize that physalins may also regulate osteoclast functions.

In this study, we predicted the possible biological functions of physalin A, B, D, and F by using BATMAN-TCM, an in silico bioinformatics analysis tool for the molecular mechanism of TCM (14). Based on the in silico results, the activity of physalins A, B, D and F against RANKL-induced osteoclastogenesis was evaluated in vitro and in vivo with exploration of detailed underlying molecular mechanisms.

ISSN: 1976-670X (electronic edition)

Copyright (c) 2020 by the The Korean Society for Biochemistry and Molecular Biology

(ㄷ) This is an open-access article distributed under the terms of the Creative Commons Attribution Non-Commercial License (http://creativecommons.org/licenses/by-nc/4.0) which permits unrestricted non-commercial use, distribution, and reproduction in any medium, provided the original work is properly cited. 


\section{RESULTS}

\section{In silico prediction of physalin A, B, D, and F biological functions}

The chemical formulas of physalin A, B, D and $F$ are shown in Supplementary Fig. S1A. We first used the BATMAN-TCM system to predict the potential biological functions of different physalins. Significantly enriched KEGG pathways, Gene Ontology (GO) terms, and OMIM/TTD disease phenotypes, among the query potential targets together with corresponding adjusted $\mathrm{P}$ values and targets mapped to this term of different physalins, are showed in the supplementary documents. Ten of the most enriched $\mathrm{GO}$ terms of each physalin are shown in Supplementary Fig. S1B. Because of their similar chemical constructions, all four physalins analyzed share common enriched GO terms such as "Protein Binding, Bridging" and "Cell-Cell Signaling", suggesting the similar biological effects they might have in common. Furthermore, the visualization of the molecular pathway and disease-correlation networks of the four physalins are shown in Supplementary Fig. S2-S5. The in silico results suggested that the physalin family members have similar biological functions, but the details are not exactly the same. Moreover, their cytotoxicity cannot be seen from the prediction.

\section{Evaluation of physalin toxicity in BMMs in the presence of RANKL}

BMMs are the most important osteoclast precursor pool in mammals. To study the effects of physalins on osteoclastogenesis, we first evaluated the cytotoxicity of physalins in BMMs treated with RANKL. To investigate the effects of physalins on cell apoptosis, we used flow cytometry to test the FITC-Annexin-V/PI stain (Supplementary Fig. S6A). BMMs (in the presence of RANKL) were treated with physalins $A, B, D$, and F with different concentrations $(0,10 \mu \mathrm{M}, 30 \mu \mathrm{M}, 50 \mu \mathrm{M})$. Quantification analysis showed that both the early and the late cell apoptosis rate significantly increased at the concentration of $10 \mu \mathrm{M}$ for both physalins (Supplementary Fig. S6B). We then investigated the effects of physalin $A, B, D$ and $F$ on the cell viability of BMMs. We found that physalin $A, D$, and $F$ began to decrease BMM cell viability significantly when the dosage reached $30 \mu \mathrm{M}$. For physalin $\mathrm{B}$, the concentration of $40 \mu \mathrm{M}$ started to show significant inhibitory effects on BMM cell viability (Supplementary Fig. S6C).

\section{Evaluation of physalin inhibitory effects on RANKL-induced osteoclastogenesis}

To further evaluate the effects of physalins (A, B, D and F) on RANKL-induced osteoclastogenesis in vitro, we isolated BMMs from mouse bone marrow and induced them with RANKL $(100 \mathrm{ng} / \mathrm{ml})$ and M-CSF $(50 \mathrm{ng} / \mathrm{ml})$ for 5 days to obtain mature osteoclasts. Physalin A, B, D, and F with different concentrations $(0,2 \mu \mathrm{M}, 4 \mu \mathrm{M}, 6 \mu \mathrm{M}, 8 \mu \mathrm{M}, 10 \mu \mathrm{M})$ were introduced together with RANKL and M-CSF. We used tartrate-resistant acid phosphatase (TRAP) stain after 5 day's induction to evaluate osteoclast differentiation (Fig. 1A). Quantification analysis of osteoclast number per well showed that physalin $A, B, D$, and $F$ all significantly reduced the number of osteoclast cells per well from the concentration of 8 $\mu \mathrm{M}$. The concentration was $10 \mu \mathrm{M}$ when it came to relative TRAP activity (Fig. 1B). Further quantification of the above results showed that physalin $\mathrm{D}$ showed significantly lower inhibitory concentration $50 \% \quad\left(\mathrm{IC}_{50}\right)$ than did the other physalins (A, B, and F). Although physalin B showed the highest lethal concentration $50 \%$ ( $\left.\mathrm{LC}_{50}\right)$ (no significance), the selectivity index (SI) showed that among the four physalins, physalin D is the most effective in inhibiting RANKL-induced osteoclastogenesis (Supplementary Table S1).

\section{Physalin D inhibits RANKL-induced osteoclastogenesis in vitro}

To confirm the inhibitory effects of physalin $\mathrm{D}$ on RANKL-induced osteoclastogenesis in vitro, we then evaluated its effects on osteoclast maturation and cytoskeleton formation. Immunostaining of F-actin showed that physalin D of $5 \mu \mathrm{M}$ robustly inhibited osteoclast maturation and cytoskeleton formation. Quantification analysis showed that the average nuclei number of osteoclasts decreased significantly upon physalin D administration, suggesting the blocked cell-cell fusion (Fig. 2A). In addition, we evaluated the effects of physalin $\mathrm{D}$ on osteoclastic bone resorption by a pit-formation

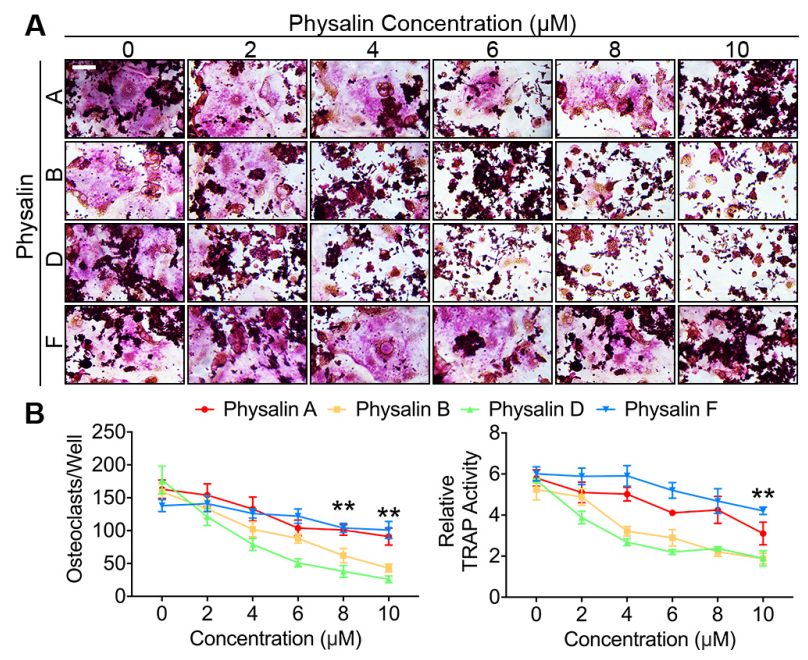

Fig. 1. Evaluation of physalin inhibitory effects on RANKL-induced osteoclastogenesis. (A) TRAP stain of RANKL-stimulated BMMs treated with physalins $(0,2 \mu \mathrm{M}, 4 \mu \mathrm{M}, 6 \mu \mathrm{M}, 8 \mu \mathrm{M}, 10 \mu \mathrm{M})$. Bar represents $200 \mu \mathrm{m}$; (B) Quantification of TRAP+ osteoclast number per well and relative TRAP activity. Images are representative of three independent experiments. The data in the figures represent the averages \pm SD. Significant differences are indicated as $* * \mathrm{P}<0.01$. 


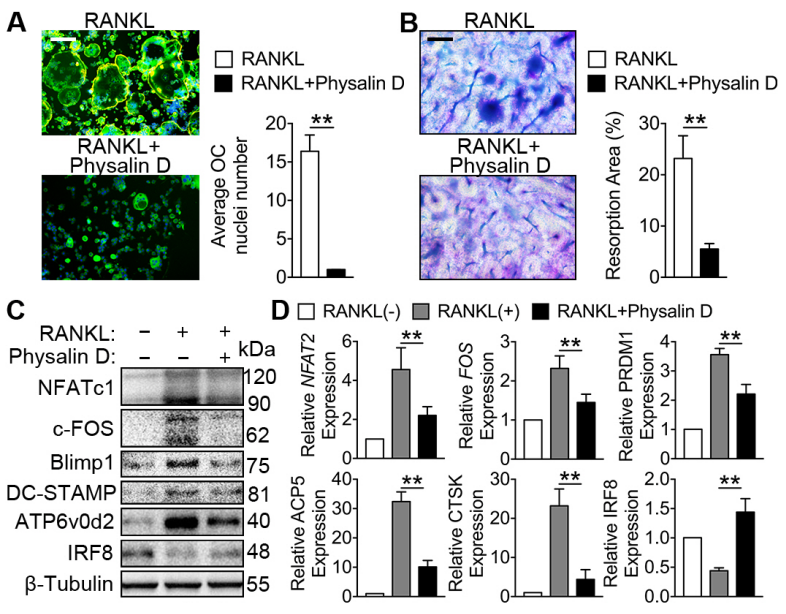

Fig. 2. Physalin D inhibits RANKL-induced osteoclastogenesis in vitro. (A) Immunostaining of F-actin in RANKL-stimulated BMMs treated with physalin D $(5 \mu M)$. Bar represents $200 \mu \mathrm{m}$. Quantification of average osteoclast nuclei number is shown on the right; (B) Representative pit-formation assay images on bovine bone slices. Bar represents $200 \mu \mathrm{m}$. Quantification of resorption area is shown on the right; (C) Western blot analysis of NFATc1, c-FOS, Blimp1, DC-STAMP, ATP6v0d2, IRF8, and $\beta$-Tubulin in RANKL-stimulated BMMs treated with physalin D $(5 \mu \mathrm{M})$; (D) Relative mRNA expression of NFAT2, FOS, PRDM1, ACP5, CTSK, and IRF8 in RANKL-stimulated BMMs treated with physalin D (5 $\mu \mathrm{M})$ after $24 \mathrm{~h}$. Images are representative of three independent experiments. The data in the figures represent the averages \pm SD. Significant differences are indicated as $* * P<0.01$.

assay using bovine bone slices. We found that physalin D of 5 $\mu \mathrm{M}$ significantly decreased the bone resorption of osteoclasts, suggesting a reduced osteoclast function (Fig. 2B). We further evaluated the expressions of several important transcription factors and fusogenic molecules on the protein level during osteoclastogenesis. We found that positive regulator NFATc1, c-FOS, B-Lymphocyte-Induced Maturation Protein (Blimp) 1, fusogenic molecules Dendrocyte Expressed Seven Transmembrane Protein (DC-STAMP), and ATPase $\mathrm{H}+$ Transporting V0 Subunit D2 (ATP6v0d2) were all downregulated by physalin D $(5 \mu \mathrm{M})$ treatment on day 3 after RANKL induction (Fig. 2C). On the mRNA level, the expression of NFATc1 (NFAT2), c-FOS (FOS), Blimp1 (PRDM1), and DC-STAMP (TM7SF4) at $24 \mathrm{~h}$ was consistently downregulated by physalin $\mathrm{D}$ treatment together with OC marker ACP5 and CTSK, whereas the ATP6v0d2 (ATP6v0d2) showed no obvious change (Fig. 2D, Supplemental Fig. S7). It is interesting to notice that negative regulator Interferon Regulatory Factor 8 (IRF8) was upregulated by physalin $\mathrm{D}$ treatment on both the protein level and the mRNA level. Overall, we concluded that physalin D effectively inhibited RANKL-induced osteoclastogenesis in vitro.
A
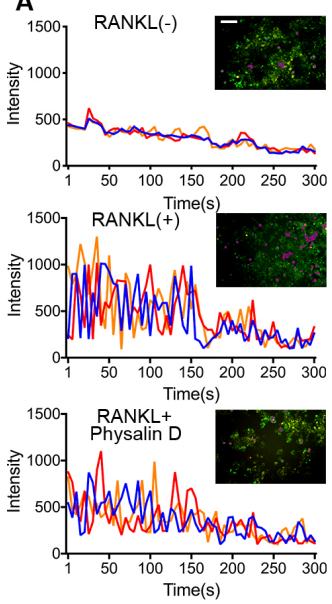

B

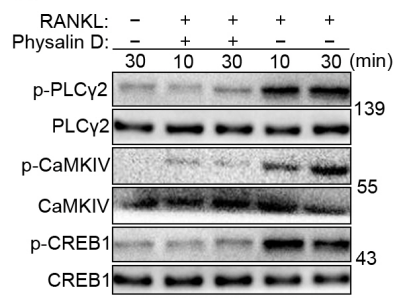

C

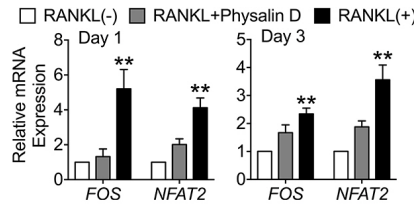

Fig. 3. Physalin D suppresses the PLC $\gamma 2-C a M K-C R E B$ pathway during osteoclastogenesis. (A) Fluo-4 fluorescent images of BMMs treated with physalin D $(5 \mu \mathrm{M})$ upon RANKL stimulation. Pseudo-color labeled (purple) area represents actively sparkling cells, bar represents $400 \mu \mathrm{m}$. Three representative traces are presented; fluorescence ratio change was recorded every $5 \mathrm{~s}$ for $300 \mathrm{~s}$; (B) Western blot analysis of p-PLC $\gamma 2$, PLC $\gamma 2$, p-CaMKIV, CaMKIV, p-CREB, and CREB in RANKL-stimulated BMMs treated with physalin D $(5 \mu \mathrm{M})$; (C) Relative mRNA expression of NFAT2 and FOS in RANKL-stimulated BMMs treated with physalin D $(5 \mu \mathrm{M})$ on day 1 and day 3 . Images are representative of three independent experiments. The data in the figures represent the averages \pm SD. Significant differences are indicated as $* * P<0.01$ ).

\section{Physalin D suppresses the PLC $\gamma 2$-CaMK-CREB pathway during osteoclastogenesis}

To understand the details of the underlying mechanisms by which physalin D inhibits RANKL-induced osteoclastogenesis, we focused on the calcium signaling, which is crucial for osteoclast differentiation and maturation (15). NFATc1 is activated by intracellular calcium $\left(\left[\mathrm{Ca}\left(2^{+}\right)\right](\mathrm{i})\right)$ oscillation upon RANKL stimulation. We first detected the effects of physalin D on $\left[\mathrm{Ca}\left(2^{+}\right)\right](\mathrm{i})$ oscillation. We found that both average amplitude and frequency of $\left[\mathrm{Ca}\left(2^{+}\right)\right](\mathrm{i})$ oscillation were increased upon RANKL stimulation (1s-300s) compared with BMMs without RANKL treatment. Introduction of physalin D robustly alleviated $\left[\mathrm{Ca}\left(2^{+}\right)\right](\mathrm{i})$ oscillation stimulated by RANKL (Fig. 3A, Supplementary Table 2-5). RANKL-RANK signaling activates phospholipase $\mathrm{C} \gamma 2$ (PLC $\gamma 2)$ and leads to an increase in $\left[\mathrm{Ca}\left(2^{+}\right)\right](\mathrm{i})$ via ITAM-harboring molecules DAP12 and FCR $\gamma$, followed by activation of $\mathrm{Ca}^{2+} /$ calmodulin-dependent protein kinases (CaMK)IV, which mainly contribute to activating cAMP-responsive element binding protein (CREB) $(16,17)$. We found that phosphorylation of PLC $\gamma 2$, CaMKIV and CREB by RANKL were significantly suppressed by physalin $D$ treatment $(5 \mu \mathrm{M})$ in BMMs without a substantial change in total protein expression (Fig. 3B). In contrast, downstream FOS and NFAT2 expressions on day 1 and day 3 were also decreased by 
physalin D treatment $(5 \mu \mathrm{M})$.

\section{Physalin D protects SRANKL induced bone loss}

We then evaluated the in vivo effects of physalin $\mathrm{D}$ in bone metabolism using a model of rapid bone loss induced by soluble RANKL (sRANKL) (18). Mice were injected intraperitoneally at 24-h intervals for 3 days, followed by daily administration of physalin D for another 17 days (Fig. 4A). After sacrificing the mice on day 21 , we did $\mu \mathrm{CT}$ analysis using dissected femurs (Fig. 4B). Quantification analysis revealed that the model of SRANKL-induced bone loss was successful. The decrease of BMD, BV/TV, Tb.N, and Tb.Th,

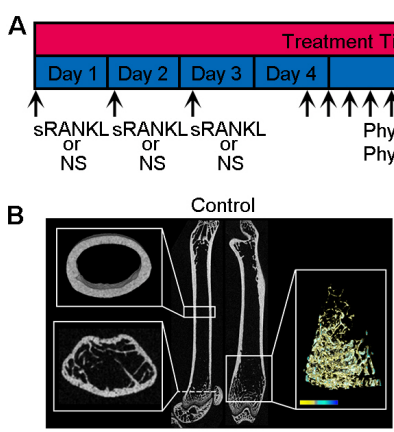

Physalin D (low)

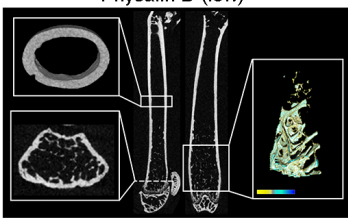

C.

○ Control • sRANKL • Physalin D (low) $\triangle$ Physalin D (high)
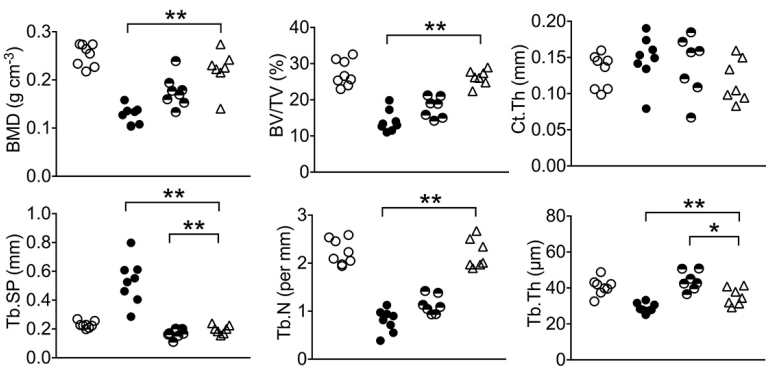

Fig. 4. Physalin D protects sRANKL-induced bone loss. (A) The experimental design of the sRANKL-induced bone-loss model and following intervention strategy. (B) Representative $\mu \mathrm{CT}$ images of longitudinal section femurs of mice, cross-sectional view of the distal femurs, cortical bone, and reconstructed trabecular structure of the ROI (white solid line box) ( $n=8)$. Color scale bar represents bone mineral density level. (C) Quantitative $\mu \mathrm{CT}$ analysis of bone mineral density (BMD), trabecular bone volume fraction (BV/TV), cortical bone thickness (Ct. Th), trabecular number $(\mathrm{Tb} . \mathrm{N})$, trabecular separation $(\mathrm{Tb} . \mathrm{Sp})$, and trabecular thickness (Tb. Th) $(n=8)$. The data in the figures represent the averages \pm SD. Significant differences are indicated as $* P<$ 0.05 or $* * \mathrm{P}<0.01$ and the increase of Tb.Sp were significantly restored by high-dosage treatments of physalin D (Fig. 4C). Moreover, evaluation of TRAP-positive osteoclast surface proportion was also consistent with microCT results, suggesting an inhibitory effect of physalin D in vivo (Supplemental Fig. S8). These in vivo results revealed that physalin $\mathrm{D}$ can inhibit bone loss caused by excessive osteoclastic activity.

\section{DISCUSSION}

A recent study reported that physalin B has a relative long dwell time with a half-life of $321.2 \pm 29.5 \mathrm{~min}$ and clearance of $175.4 \pm 25.7 \mathrm{ml} / \mathrm{min} / \mathrm{kg}$ in rats after intravenous injection (19). Although widely distributed in tissues, physalin B penetration was particularly high in the lungs. Another more recent study suggested that orally administered physalin A can further be metabolized to two sulphonate metabolites characterized from the feces (20). Nevertheless, the detailed function of these metabolites remains unknown. More in vivo studies like this about the other physalins are strongly encouraged before efficient animal studies. Physalins have been reported to have proapoptotic effects in cancer cells. Physalin B was shown pro-apoptotic in melanoma cancer-cell lines via activating the NOXA-related pathway instead of normal cells (21). The reasons for this discrepancy between tumor cells and normal cells were not discussed; however, their discovery is somewhat similar to previous reports (22). In our study, we also found that physalins induce cell apoptosis in BMMs. However, the $\mathrm{IC}_{50}$ values of physalins are much lower than the $\mathrm{LC}_{50}$ values suggesting that the inhibitory effects of physalins on RANKL-induced osteoclastogenesis is not though cytotoxicity.

We found that physalin D can regulate the calcium signaling during RANKL-induced osteoclastogenesis. Calcium ions $\left(\mathrm{Ca} 2^{+}\right)$affect nearly every aspect of cellular life; they also play a crucial role during osteoclastogenesis. Cytoplasmic $\mathrm{Ca}^{+}$ oscillations in RANKL-induced osteoclast differentiation provide a digital $\mathrm{Ca}_{2}{ }^{+}$signal that stimulates osteoclasts to upregulate and autoamplify the expression of NFATc1 (2). RANKL introduction induced a continuing intracellular $\mathrm{Ca} 2^{+}$ increase in osteoclasts cultured from human monocytes. However, the extracellular $\mathrm{Ca}_{2}{ }^{+}$influx seemed to be the main source for the $\mathrm{Ca}^{+}$flux (23). It has also been shown that the CaMKIV-CREB pathway is critical for osteoclastogenesis (24). Inhibition of CaMKs as well as the genetically ablation of Camk4 decreased the phosphorylation of CREB and resulted in downregulation of c-Fos and NFATc1. However, studies also showed that RANKL-induced $\left[\mathrm{Ca}\left(2^{+}\right)\right]$(i) increase can inhibit cell motility and the bone-resorption activity of mature osteoclasts (25). We found that physalin D inhibits phosphorylation of PLC 2 , thus attenuating the RANKL-induced $\left[\mathrm{Ca}\left(2^{+}\right)\right](\mathrm{i})$ oscillation and the downstream CaMK-CREB pathway. The regulating effects of physalin $\mathrm{D}$ in calcium signaling still needs to be confirmed in other cell types. It is 
worth mentioning that, although the SRANKL rapid bone-loss model could facilitate preclinical work in the discovery of drug candidates for the treatment of osteoporosis, there are still limitations, especially in simulating the estrogen-related bone loss.

In this study, we looked at the in vitro effects of physalin A, $B, D$, and $F$ on RANKL-induced osteoclastogenesis from primary BMMs. We found that all four physalins have inhibitory effects on RANKL-induced osteoclastogenesis; however, combining the $\mathrm{IC}_{50}$ and $\mathrm{LC}_{50}$ results, physalin $\mathrm{D}$ has the best efficacy in inhibiting osteoclastogenesis. The inhibitory effects of physalin $\mathrm{D}$ were then confirmed by immunostaining for cytoskeletal observation and a pit-formation assay for osteoclast function evaluation. On the molecular level, we found that physalin D suppressed the calcium signaling during RANKL-induced osteoclastogenesis. $\left[\mathrm{Ca}\left(2^{+}\right)\right](\mathrm{i})$ oscillation was attenuated, and phosphorylation of PLC $\gamma 2$, CaMK, and CREB were inhibited. An animal study showed that physalin D protects SRANKLinduced bone loss. Taken together, our results suggested that physalin D inhibits RANKL-induced osteoclastogenesis and bone loss by suppressing the PLC 2 2-CaMK-CREB pathway.

\section{MATERIALS AND METHODS}

\section{Mice}

We purchased female C57BL/6 mice from the animal center of Army Medical University. All procedures involving mice and experimental protocols were approved by the Institutional Animal Care and Use Committee of the Army Medical University, and were performed according to the guidelines on laboratory animal care and use. We tried to reduce the suffering of the animals as much as possible. We established a model of sRANKL-induced rapid bone loss by injecting sRANKL $(2 \mathrm{mg} / \mathrm{kg}$ ) intraperitoneally at $24 \mathrm{~h}$ intervals for 3 days into 7-wk-old female mice. Physalin D was intraperitoneally injected with low $(10 \mathrm{mg} / \mathrm{kg})$ and high $(100 \mathrm{mg} / \mathrm{kg})$ doses daily for 2 weeks. Bone-marrow cells were separated and cultured with M-CSF (50 ng/ml) for $72 \mathrm{~h}$ to obtain bone-marrow macrophages (BMMs), which were cultured in $\alpha$-minimal essential medium (MEM) containing 10\% $\mathrm{FBS}$ and $1 \%$ Penicillin-streptomycin solution.

\section{In vitro assays for osteoclast differentiation, fusion, and function}

BMMs and preosteoclasts were cultured in $\alpha$-MEM containing $10 \%$ FBS. For TRAP staining, cells were cultured in a 96-well plate at a density of $5 \times 10^{3}$ cells per well with RANKL (100 $\mathrm{ng} / \mathrm{ml})$ and M-CSF $(50 \mathrm{ng} / \mathrm{ml})$ for 5 days. Cells were then fixed in preheated $4 \%$ paraformaldehyde (PFA) of $37^{\circ} \mathrm{C}$ for $2 \mathrm{~min}$ and then stained with TRAP staining solutions according to the manufacturers' instructions. Mature osteoclast was counted as TRAP positive with 3 or more nuclei for identification. We measured relative TRAP activity by colorimetric analysis using software Image J2. For actin cytoskeleton and focal adhesion stain, cells were cultured on glass sheet in 48-well plates at a density of $1 \times 10^{4}$ cells/well. Cells were stimulated with M-CSF $(50 \mathrm{ng} / \mathrm{ml})$ and RANKL $(100 \mathrm{ng} / \mathrm{ml})$ for 5 days for osteoclastogenesis. Detailed procedures were described in a previous study (26). For bone resorption pit-formation tests, cells were incubated in 48-well plates covered with bovine bone slices of $1 \times 10^{4}$ cells/well, as described previously (27). In brief, cells were induced with RANKL (100 ng/ml) and M-CSF $(50 \mathrm{ng} / \mathrm{ml})$ for 3 days. Bleach solution was added to remove cells followed by light microscope observation.

\section{$\mu \mathrm{CT}$ analysis}

We used a Bruker MicroCT Skyscan 1272 system (Kontich, Belgium) for $\mu \mathrm{CT}$ analysis. Isotropic voxel size of $10.0 \mu \mathrm{m}$ was used to scan the whole femur. We did scans in $4 \%$ paraformaldehyde and used an x-ray tube potential of $60 \mathrm{kV}$, an x-ray intensity of $166 \mu \mathrm{A}$, and an exposure time of 1700 $\mathrm{ms}$. For distal femur trabecular bone analysis, a 3-mm region started at $0.8 \mathrm{~mm}$ proximal to the central epiphysis of the femur was contoured. For femur cortical bone analysis, a 0.5 -mm region was started at $4.5 \mathrm{~mm}$ proximal to the central epiphysis of the femur. Trabecular and cortical bones were thresholded at 86-255 (8-bit grey-scale bitmap). Reconstruction was accomplished by Nrecon (Ver. 1.6.10); 3D images were obtained from contoured 2D images by methods based on distance transformation of the original grey-scale images (CTvox, Ver. 3.0.0). We did 3D and 2D analysis using software CT Analyser (Ver. 1.15.4.0).

\section{Statistics}

All data presented are representative of three or more independent assays performed in triplicate unless otherwise indicated. All data in the figures are presented as mean \pm SD unless otherwise indicated. One-way ANOVA was used with later on Newman-Keuls methods to evaluate the significance of result differences. Significance was regarded as $* \mathrm{P}<0.05$, and ${ }^{* * P}<0.01$. The detailed methods are shown in the supplemental materials.

\section{ACKNOWLEDGEMENTS}

This work was supported by a grant from the Nature Science Foundation of China (NSFC) (No.81802166), Nature Science Foundation of Liaoning Province (20170540938, 2019-BS-264), and TMMU funding for young investigators (2017MPRC-04).

\section{CONFLICTS OF INTEREST}

The authors have no conflicting interests.

\section{REFERENCES}

1. Yasuda H, Shima N, Nakagawa N et al (1998) Osteoclast differentiation factor is a ligand for osteoprotegerin/ 
osteoclastogenesis-inhibitory factor and is identical to TRANCE/RANKL. Proc Nat Acad Sci U S A 95, 3597-3602

2. Hwang SY and Putney JW Jr (2011) Calcium signaling in osteoclasts. Biochim Biophys Acta 1813, 979-983

3. Li P, Liu C, Hu M, Long M, Zhang D and Huo B (2014) Fluid flow-induced calcium response in osteoclasts: signaling pathways. Ann Biomed Eng 42, 1250-1260

4. Hadjidakis DJ and Androulakis II (2006) Bone remodeling. Ann New York Acad Sci 1092, 385-396

5. Rodan GA and Martin TJ (2000) Therapeutic approaches to bone diseases. Science 289, 1508-1514

6. Bastos GN, Silveira AJ, Salgado CG, Picanco-Diniz DL and do Nascimento JL (2008) Physalis angulata extract exerts anti-inflammatory effects in rats by inhibiting different pathways. J Ethnopharmacol 118, 246-251

7. Soares MB, Bellintani MC, Ribeiro IM, Tomassini TC and Ribeiro dos Santos R (2003) Inhibition of macrophage activation and lipopolysaccaride-induced death by secosteroids purified from Physalis angulata L. Eur J Pharmacol 459, 107-112

8. Pinto LA, Meira CS, Villarreal CF et al (2016) Physalin F, a seco-steroid from Physalis angulata L., has immunosuppressive activity in peripheral blood mononuclear cells from patients with HTLV1-associated myelopathy. Biomed Pharmacother 79, 129-134

9. Yu Y, Sun L, Ma L, Li J, Hu L and Liu J (2010) Investigation of the immunosuppressive activity of Physalin $\mathrm{H}$ on $\mathrm{T}$ lymphocytes. Int Immunopharmacol 10, 290-297

10. Jacobo-Herrera NJ, Bremner $P$, Marquez $N$ et al (2006) Physalins from Witheringia solanacea as modulators of the NF-kappaB cascade. J Nat Prod 69, 328-331

11. Sa MS, de Menezes MN, Krettli AU et al (2011) Antimalarial activity of physalins B, D, F, and G. J Nat Prod 74, 2269-2272

12. Yang YJ, Yi L, Wang Q, Xie BB, Dong $Y$ and Sha CW (2017) Anti-inflammatory effects of physalin $E$ from Physalis angulata on lipopolysaccharide-stimulated RAW 264.7 cells through inhibition of NF-kappaB pathway. Immunopharmacol Immunotoxicol 39, 74-79

13. Ding N, Wang Y, Dou C et al (2019) Physalin D regulates macrophage M1/M2 polarization via the STAT1/6 pathway. J Cell Physiol 234, 8788-8796

14. Liu Z, Guo F, Wang Y et al (2016) BATMAN-TCM: a Bioinformatics Analysis Tool for Molecular mechANism of Traditional Chinese Medicine. Sci Rep 6, 21146

15. Kim H, Kim T, Jeong BC et al (2013) Tmem64 modulates calcium signaling during RANKL-mediated osteoclast differentiation. Cell Metab 17, 249-260

16. Takayanagi $\mathrm{H}$ (2007) Osteoimmunology: shared mechanisms and crosstalk between the immune and bone systems. Nature reviews. Immunology 7, 292-304

17. Wada $T$, Nakashima $T$, Hiroshi $N$ and Penninger JM (2006) RANKL-RANK signaling in osteoclastogenesis and bone disease. Trends Mol Med 12, 17-25

18. Tomimori Y, Mori K, Koide M et al (2009) Evaluation of pharmaceuticals with a novel 50-hour animal model of bone loss. J Bones Miner Res 24, 1194-1205

19. Zheng Y, Chen J, Liu L, Liang $X$ and Hong D (2016) In vivo pharmacokinetics of and tissue distribution study of physalin B after intravenous administration in rats by liquid chromatography with tandem mass spectrometry. Biomed Chromatogr 30, 1278-1284

20. Liu H, Wang K, Xia G et al (2018) Two sulfonate metabolites of physalin $A$ in rats. Xenobiotica 48, 11-17

21. Hsu CC, Wu YC, Farh L et al (2012) Physalin B from Physalis angulata triggers the NOXA-related apoptosis pathway of human melanoma A375 cells. Food Chem Toxicol 50, 619-624

22. Vandenberghe I, Creancier L, Vispe $S$ et al (2008) Physalin $B$, a novel inhibitor of the ubiquitin-proteasome pathway, triggers NOXA-associated apoptosis. Biochem Pharmacol $76,453-462$

23. Chamoux E, Bisson M, Payet MD and Roux S (2010) TRPV5 mediates a receptor activator of NF-kappaB (RANK) ligandinduced increase in cytosolic $\mathrm{Ca} 2+$ in human osteoclasts and down-regulates bone resorption. J Biol Chem 285, 25354-25362

24. Sato K, Suematsu A, Nakashima T et al (2006) Regulation of osteoclast differentiation and function by the CaMK-CREB pathway. Nat Med 12, 1410-1416

25. Kajiya H (2012) Calcium signaling in osteoclast differentiation and bone resorption. Adv Exp Med Biol 740, 917932

26. Dou C, Zhang C, Kang F et al (2014) MiR-7b directly targets DC-STAMP causing suppression of NFATC1 and c-Fos signaling during osteoclast fusion and differentiation. Biochim Biophys Acta 1839, 1084-1096

27. Dou C, Ding N, Xing J et al (2016) Dihydroartemisinin attenuates lipopolysaccharide-induced osteoclastogenesis and bone loss via the mitochondria-dependent apoptosis pathway. Cell Death Dis 7, e2162 